\title{
Una lectura comparativa sobre el nivel de satisfacción laboral de los docentes venezolanos entre 1996 y $2018^{1}$
}

\author{
A comparative reading on the level of job satisfaction \\ of Venezuelan teachers between 1996 y 2018 \\ Uma leitura comparativa sobre o nível de satisfação laboral \\ dos docentes venezuelanos entre 1996 e 2018
}

Tulio Ramírez
Universidad Católica Andrés Bello
Universidad Central de Venezuela

Grupo de investigación Lenguajes en Educación (LEEN)

https://orcid.org/0000-0002-9012-8707

Doris Lilia Torres $\mathrm{Cruz}^{3}$

Universidad Pedagógica y Tecnológica de Colombia Grupo de investigación Lenguajes en Educación (LEEN) https://orcid.org/0000-0001-7234-3166

Recepción: 15/07/2019

Evaluación: 18/05/2020

Aceptación: 28/05/2020

Artículo de Investigación

https://doi.org/10.19053/01227238.11969

\section{RESUMEN}

El objetivo de esta investigación es comparar los niveles de satisfacción laboral de una muestra de docentes venezolanos de educación primaria y bachillerato del sector oficial, entre los años de 1996 y 2006. Originalidad: Se evidenciaron altos niveles de insatisfacción laboral en los siete factores que cargan la escala aplicada, cuyos resultados se compararon con los obtenidos entre 1996 y 2006 y se observó una profundización del estado de insatisfacción que hace 24 años ya se percibía, sobre todo en los factores extrínsecos al oficio, como remuneraciones, ambiente de trabajo y sus condiciones, así como con los beneficios. Método: se consideró la investigación de enfoque mixto, con la cual se utiliza la estrategia de la escala de satisfacción laboral, administrada de manera on line y construida desde la Teoría Bifactorial elaborada por Herzberg, Mausner y

1 Este artículo de investigación hace parte del proyecto 1988, registrado en la Dirección de Investigaciones de la Universidad Pedagógica y Tecnológica de Colombia.

2 Tulio Ramírez, sociólogo, abogado, máster en Formación de Recursos Humanos, doctor en Educación, profesor titular de la Universidad Central de Venezuela (UCV) y de la Universidad Católica Andrés Bello (UCAB). Director del Doctorado y el Postdoctorado en Educación de la UCAB. Integrante del grupo de investigación Lenguajes en Educación (LEEN-UPTC), clasificado en Colciencias según convocatoria 833 de 2018, grupo.leen@uptc.edu.co

3 Docente de tiempo completo de la Universidad Pedagógica y Tecnológica de Colombia. Coordinadora de la Cátedra UNESCO para la Lectura y Escritura en América Latina-subsede UPTC y del grupo de investigación Lenguajes en Educación (LEEN-UPTC), clasificado en Colciencias según convocatoria 833 de 2018, grupo.leen@uptc.edu.co 
Snyderman en 1959. La conclusión resalta que los factores intrínsecos o motivadores que tienen que ver con la naturaleza misma del trabajo (reconocimiento de la labor, autonomía profesional y trabajo en sí mismo) presentan niveles de satisfacción muy por debajo de los encontrados en los estudios previos referidos.

Palabras clave: magisterio venezolano; satisfacción laboral; maestros; teoría bifactorial

\section{ABSTRACT}

The research aims at comparing the levels of job satisfaction of a sample of Venezuelan official teachers of Primary and High School during the years of 1996 and 2006. Originality: High levels of job dissatisfaction were evidenced in the 7 factors that load the scale applied. Comparing these results with those achieved in 1996 and 2006, there was a deepening of the state of dissatisfaction which particularly had already been perceived since 24 years ago in extrinsic factors to work such as remuneration, the job environment and its conditions, as well as with profits. Method: a mixed approach research has been considered, with which the strategy of the job satisfaction scale is used, managed in online way and built from the Bifactorial Theory elaborated by Herzberg, Mausner and Snyderman in 1959. The conclusion highlights that the intrinsic or motivating factors that have to do with the nature of the work itself (recognition of work, professional autonomy and work in itself) present satisfaction levels as well as those found in the previous studies referred to. Key words: Venezuelan teaching; job satisfaction; teachers; bifactorial theory

\section{RESUMO}

O objetivo desta pesquisa é comparar os níveis de satisfação laboral de uma amostra de docentes oficiais, venezuelanos de Educação Primária e Ensino Médio, durante os anos de 1996 a 2006. Originalidade: se evidenciaram altos níveis de insatisfação laboral nos 7 fatores que compõem a escala aplicada. Ao comparar estes resultados com os obtidos em 1996 e 2006, se observou um aprofundamento do estado de insatisfação, que já se percebia há 24 anos, sobretudo nos fatores intrínsecos ao trabalho como as remunerações, o ambiente de trabalho e suas condições, assim como os benefícios. Método: considera-se a pesquisa de enfoque misto, com o qual se utiliza a estratégia da escala de satisfação laboral, administrada de maneira on line e construída a partir da Teoria Bifatorial elaborada por Herzberg, Mausner e Snyderman em 1959. A conclusão ressalta que os fatores intrínsecos ou motivadores que têm relação com a natureza mesma do trabalho (reconhecimento do labor, autonomia profissional e trabalho em si mesmo) apresentam níveis de satisfação muito abaixo dos encontrados nos estudos prévios referidos.

Palavras-chave: Magistério venezoelano; satisfação laboral; docentes; Teoria Bifatorial.

\section{INTRODUCCIÓN}

La profesión docente en América Latina se ha caracterizado por ser una de las actividades peor remuneradas en comparación con el estándar de remuneraciones para otras profesiones universitarias. A pesar de que desde mediados del siglo XX en la mayoría de los países de la región es requisito haber obtenido 
el título de educación superior para optar por un cargo docente; tal exigencia no ha traído como consecuencia un aumento en las remuneraciones y beneficios a la par de otras actividades profesionales. Los gobiernos han preferido enfrentar las presiones de los gremios docentes antes de ceder a las peticiones de mejoras salariales y ampliación de los beneficios socioeconómicos.

El caso venezolano no ha sido diferente. Las tensiones entre los maestros y sus gremios y el gobierno nacional han sido recurrentes. A pesar de que cada dos o tres años se firman convenciones colectivas que pretenden mejorar las condiciones anteriores, la constante es que los diferentes gobiernos hacen caso omiso a estos compromisos, con lo cual se generan reclamos, huelgas y actividades sindicales que son contrarrestadas por las autoridades educativas con amenazas de despidos masivos o simplemente haciendo oídos sordos ante tales reclamos. A pesar de los bajos salarios y peores condiciones de trabajo, en Venezuela no se han evidenciado altos niveles de rotación laboral ${ }^{4}$

Los años que van de 1995 a 1998 fueron para Venezuela escenario de muchos conflictos laborales. Los altos índices de inflación y la cada vez menor disponibilidad de recursos económicos por parte del Estado debido a que los precios del petróleo venían descendiendo por el aumento de la oferta del crudo en el mundo, generaron una coyuntura caracterizada por una abierta tensión entre los trabajadores y el gobierno nacional. El Estado venezolano, cada vez con menos recursos, difícilmente podía cubrir las expectativas de aumento salarial para el funcionariado de la administración pública y lo mismo sucedía en el resto de la economía. Las demandas salariales llevaron a momentos de tensa conflictividad en el mundo laboral, realidad de la que no pudo escapar el campo magisterial.

Eran los años de la Comisión Tripartita, instancia creada por el gobierno del presidente Rafael Caldera y conformada con representación del gobierno nacional a través del Ministerio de Planificación, de los trabajadores a través de las centrales sindicales más importantes del país y del sector empresarial. Su cometido era llegar a acuerdos sobre temas como salarios, prestaciones sociales, y la estructura de precios de los productos de primera necesidad. En ese contexto se desencadenaron huelgas magisteriales que presionaban para una mejora del poder adquisitivo de maestros y profesores.

En medio de ese escenario de claro descontento social nos propusimos realizar un estudio sobre los niveles de satisfacción laboral de los maestros venezolanos. Sus resultados arrojaron cifras que revelaban que, si bien había mucha insatisfacción con las remuneraciones, los beneficios sociolaborales, las condiciones de trabajo y otros aspectos que señalaremos más adelante, los docentes se sentían muy satisfechos con el trabajo en sí mismo y la autonomía profesional de la cual gozaban para el desempeño de su tarea docente.

Diez años después (en 2006), durante el gobierno del teniente coronel Hugo Chávez Frías, nos planteamos replicar este mismo estudio. Nuestra inquietud

4 Tulio Ramírez, María E. D’Aubeterre y Juan C. Álvarez, “Un estudio sobre estrés laboral en una muestra de maestros de Educación Básica del área metropolitana de Caracas", ExtraMuros, n. 29 (2009); "Percepción de estresores en profesores universitarios venezolanos. Un estudio descriptivo", Akademus vol. 14, n. ${ }^{\circ s} 1$ y 2 (2012). 
giraba en torno a la curiosidad de conocer qué tanto habían variado los resultados del estudio de 1996, dado que Venezuela se encontraba con una situación política y económica diferente a la de diez años atrás. Era una época de cierta bonanza económica debido al alza de los precios del petróleo, pero de mucha conflictividad política. Unos años antes (en 2002) se había desarrollado el llamado "Paro petrolero" que costó el despido de más de dieciocho mil trabajadores de la industria petrolera estatal PDVSA y una persecución política implacable a todo el que disintiera del gobierno. Los maestros durante ese período fueron amenazados con despidos de comprobarse su adhesión al paro cívico, para ser sustituidos por maestros afectos al gobierno bajo la figura de docentes interinos. Poco antes (año 2001) se había implementado el Decreto 1.011, que suponía la creación de "supervisores itinerantes" con la potestad de sancionar a directivos y docentes que no cumplieran los "dictados del Ministerio de Educación". En el año 2006 se dio el primer intento de implantar un diseño curricular para la "educación bolivariana", cargado de sesgos ideológicos tan evidentes que fue rechazado públicamente por docentes, gremios, universidades y ONG interesadas en el área educativa.

Los resultados obtenidos a través de ese estudio del año 2006 no solo corroboraron el descontento de los docentes sobre los mismos aspectos que fueron señalados en la investigación de 1996, sino se extendieron a factores que generaban satisfacción según ese estudio de diez años atrás. Nos referimos a la autonomía profesional en el trabajo docente; tal cambio de percepción era explicable. En esa época de claro acoso laboral por motivaciones políticas y de injerencia desmedida en la actividad de los docentes a través de la pretensión de imponer un diseño curricular ideologizante y rechazado por gran parte de la sociedad venezolana, los maestros y profesores fueron víctimas de una cada vez mayor intromisión en su trabajo en el aula.

Del 2006 al 2018 la situación varió un poco, pero para peor. Los niveles de hiperinflación pusieron a Venezuela en los primeros lugares de los países con mayores distorsiones económicas en el mundo, la escasez de alimentos y productos por la destrucción del aparato productivo y la imposibilidad de importar por no contar con recursos debido a la reducción de la producción petrolera y la caída de los precios del crudo, sumado a la inseguridad, la falta de medicamentos y la conflictividad política, han motivado a que repliquemos los estudios de 1996 y 2006. Los resultados se observarán a lo largo del artículo y darán luces sobre cómo han aumentado los niveles de descontento y de insatisfacción laboral después de 24 años de deterioro sostenido de la calidad de vida de los venezolanos.

Para 2018 cambiaron las condiciones en Venezuela. Para el cierre de ese año la inflación rondaba el millón por ciento, la más alta del mundo, con un salario mínimo que, para comienzos de 2019, apenas superaba los cinco dólares mensuales de acuerdo con la tasa oficial. Era de prever que los docentes tenían sobradas razones para estar insatisfechos con los llamados factores higiénicos o extrínsecos al trabajo. Las remuneraciones percibidas no alcanzaban para cubrir 
la cesta básica y las condiciones de trabajo empeoraron por la falta de recursos. La caída de la producción petrolera mermó los presupuestos para el mantenimiento de las escuelas y la dotación de los recursos que necesitaban para su funcionamiento. De igual manera, suponemos hipotéticamente, dadas las continuas presiones políticas sobre los docentes no afectos al gobierno chavista, que el descontento en esta materia se ha potenciado.

Si bien lo anterior era previsible, la incógnita siempre rondó sobre los factores motivacionales o intrínsecos al trabajo en sí mismo. En los estudios anteriores ${ }^{5}$ sobre estos factores la percepción de los docentes siempre estuvo ligada a la satisfacción, lo que explicaba, en parte, el por qué, a pesar de las desfavorables condiciones y remuneraciones, los docentes se mantenían en sus puestos de trabajo. Conocer si dicha percepción cambió después de 24 años fue lo que motivó el presente estudio.

\section{La satisfacción laboral, una preocupación de la sociedad moderna}

El desarrollo industrial no solo trajo consigo la preocupación por crear las tecnologías necesarias para hacer más eficaz y eficiente el trabajo. Si bien la expectativa de la riqueza impulsó la industria, la ciencia y la tecnología, se vio obligada a atender la salud de quienes la generaban con su fuerza de trabajo o intelecto. Así, el trabajador dejó de ser un insumo más dentro del modelo de negocios para convertirse en centro de preocupación permanente para el inversionista. Se entendió que un trabajador desmotivado e insatisfecho podía poner en peligro la inversión, toda vez que variables como calidad, eficiencia y productividad dependían en buena medida del interés y entusiasmo que aquel endosaba a sus labores.

El descubrimiento de esa simple pero no tan evidente ecuación hizo que se desarrollara en la piscología, y luego en otras disciplinas, estudios que se dirigían a identificar cuáles eran los factores del ambiente laboral o extra laboral que influían en el bienestar de las personas y, por ende, en el desempeño laboral. Se concluyó que la satisfacción o insatisfacción laboral estaban relacionadas con muchos comportamientos que pueden potenciar u obstaculizar el desarrollo de la propia persona, la organización o la sociedad ${ }^{6}$.

La insatisfacción en el trabajo ocasionada por un inadecuado ambiente genera daños a la salud física y psíquica; además, define en cada uno de los integrantes de la organización su comportamiento laboral, el cual genera un impacto diferente en cada sujeto, ocasionando expectativas laborales que se traducen en bajo rendimiento e improductividad ${ }^{7}$.

5 Ramírez, "El docente frente a su trabajo"; Ramírez y D’Aubeterre, "Los niveles de satisfacción laboral”.

6 Carlos Gamero, Análisis macroeconómico de la satisfacción laboral (Madrid: Consejo Económico y Social, 2005).

7 Carlos E.Cabrera et al., "Satisfacción laboral de profesionales de la salud en el IMSS, Jalisco 1999-2002". Revista Médica IMSS vol. 42 , n. 3 (2003). 
Desde los primeros estudios desarrollados por Hoppock ${ }^{8}$ hasta ahora son muchos los avances que se han dado con respecto al tema. Diversos autores se han dedicado a estudiar la satisfacción laboral como elemento cardinal de cualquier organización laboral en función de los resultados que espera obtener. Las derivaciones de dichos estudios han sido variadas; por una parte, se encuentran los que atribuyen la satisfacción laboral a necesidades que se encuentran distribuidas en forma de pirámide de acuerdo con la importancia e influencia que tengan en el comportamiento humano; otros sugieren que la satisfacción en el trabajo es un reflejo del grado en que una persona encuentra que su trabajo resulta deseable?.

Sin embargo, estos primeros enfoques que atribuían, de manera monocausal, los bajos niveles de rendimiento a la insatisfacción del trabajador por su trabajo, han sido superados en los últimos años por enfoques que asocian el bajo rendimiento a la insatisfacción sobre determinados factores, más que el descontento que pueda haber sobre otros. Es la tesis que han sostenido Herzberg, Mausner y Snyderman desde sus primeros estudios realizados en 1959 en trabajadores de fábricas en Estados Unidos ${ }^{10}$.

Detengámonos un poco para conocer cuáles han sido los dos principales abordajes para el tema de la satisfacción laboral. El trabajador requiere del trabajo no solo para ganarse el sustento, sino también como parte de su realización personal. De manera que las personas tratan de establecer estrategias para alinear su futuro profesional con sus perfiles personales y las exigencias de los puestos de trabajo a los que aspiran. Este proceso es lo que Merino entiende como ajuste laboral ${ }^{11}$, vale decir, el ajuste coherente entre la persona y sus expectativas de futuro y la naturaleza y exigencias del cargo. De concretarse este ajuste es probable que el trabajador se motive con lo que hace, lo haga cada vez mejor y vea compensado su esfuerzo, bien sea por la remuneración o por el reconocimiento. De no existir este ajuste es de esperarse un trabajador insatisfecho y desmotivado, lo cual ha de incidir en aspectos como la rotación, el ausentismo y la baja productividad, entre otros.

Los pioneros en los estudios sobre satisfacción laboral han identificado dos tipos de factores que inciden en los niveles de satisfacción e insatisfacción en el trabajo. Por una parte están los llamados factores higiénicos o extrínsecos que giran en torno al trabajo pero que no son el trabajo en sí mismo. Son factores como la remuneración, las condiciones de trabajo, el ambiente de trabajo, el trato justo por parte de la supervisión, la estabilidad. Vale decir, un trabajador se sentirá satisfecho si ve su esfuerzo compensado con un buen salario, condiciones óptimas de trabajo y un trato respetuoso y considerado por parte de sus supe-

8 Robert Hoppock, Job Satisfaction (New York: Harper Ed., 1935), citado en Ramírez y D’Aubeterre, “Los niveles de satisfacción laboral", 60 .

9 Victor H. Vroom, Work and Motivation (New York: Wiley, 1964), citado en Milton I. Blum y James C. Naylor, Psicología industrial: Sus fundamentos teóricos y sociales (Ciudad de México: Trillas, 1990).

10 Frederick Herzberg, Bernard Mausner y Barbara Bloch Snyderman, The Motivation To Work (New York: Wiley, 1959).

11 Enrique Merino, "Teoría del ajuste laboral y orientación vocacional", International Journal of Developmental and Educational Psychology vol. 5, n. ${ }^{\circ} 1$ (2011). 
riores. La precariedad de estos factores o su ausencia generarían altos niveles de descontento y desmotivación ${ }^{12}$. Estas teorías están inspiradas en la teoría de las necesidades humanas de Maslow ${ }^{13}$.

Otros autores como Mc Gregor ${ }^{14}$, Hackman y Lawler ${ }^{15}$, Flippo ${ }^{16}$ y Barry ${ }^{17}$, asumen que la satisfacción en el trabajo está más ligada a la autorrealización que a los beneficios externos ligados al cargo. Asumen que un trabajo retador, que amerite responsabilidad, que permite desplegar con cierta libertad la creatividad, que se realiza con respeto a la autonomía de juicio de quien lo ejerce y que es reconocido por sus superiores y sus compañeros genera mayor grado de satisfacción en el trabajador que la presencia optimizada de factores extrínsecos o higiénicos como el salario. Si bien estos autores no desprecian la importancia de los factores ambientales o extrínsecos, les dan mayor peso a los ligados al trabajo en sí mismo.

Desde una perspectiva menos unicausalista, Herzberg, Mausner y Snyderman ${ }^{18}$ asumen, a diferencia de las posiciones encontradas en cuanto a cuáles factores inciden sobre la satisfacción laboral, que ambos tipos de factores tienen una contribución diferenciada en la conformación de la satisfacción del trabajador. La llamada Teoría Bifactorial de Herzberg y colaboradores supone que existen dos tipos de factores, a saber:

Factores higiénicos o extrínsecos. Tienen que ver con aspectos que conllevan a la seguridad y estabilidad en el trabajo y se relacionan con el ambiente de trabajo, condiciones, supervisión y remuneración que se perciben; son factores del entorno del trabajo y no del trabajo en sí mismo y se llaman higiénicos porque son preventivos. En la medida en que están presentes evitan la insatisfacción del trabajador, aunque no necesariamente elevan los niveles de satisfacción por el trabajo, pero si están ausentes o precariamente presentes generan insatisfacción.

Factores motivadores o intrínsecos. Estos se refieren a la autonomía en el desempeño del trabajo, logros, reconocimiento, posibilidades de ascenso y son, por supuesto, los que generan satisfacción en el trabajador ya que tienen que ver con la autorrealización. Los citados autores descubrieron que si bien la ausencia de los factores higiénicos o extrínsecos puede generar niveles de insatisfacción, no necesariamente se traduce de manera automática en rechazo a la tarea o al cargo ya que esta puede estar cubriendo las expectativas de autorrealización profesional o personal por ser un trabajo retador, interesante, que permite decisiones autónomas en virtud de la experiencia o méritos académicos de quien las toma

12 John Perry, Las relaciones humanas en la industria (Buenos Aires: Editorial Selección Contable, 1961); Edward E. Lawler y Lyman

W. Porter, "What job attitudes tell about motivation", Harvard Business Review vol. 46, n. ${ }^{\circ} 1$ (1969); Lloyd Byars y Leslie W. Rue, Administración de recursos humanos. Conceptos y aplicaciones (México: Nueva Editorial Interamericana, 1983); Blum y Naylor, Psicología industrial.

13 Maslow, Abraham H. Motivation and Personality (New York: Harper and Row, 1954); El management según Maslow: Una visión humanista para la empresa de hoy (Barcelona: Editorial Paidós Ibérica, 2005).

14 Douglas Mc Gregor. El aspecto humano de las empresas (México: Diana, 1972).

15 Richard J. Hackman y Edward E. Lawler, "Employee reactions to job characteristics”, Journal of Applied Psychology vol. 55, n. 3 (1978).

16 Edwin B. Flippo. Principios de administración de personal (México: McGraw-Hill, 1982).

17 J. Barry, "Satisfaction in job", Cornell Review vol. 3, n. 4 (1985).

18 Herzberg, Mausner y Snyderman, The Motivation To Work. 
o que es una labor de alta estima para la sociedad (satisfacción con los factores intrínsecos).

Por el contrario, cuando los factores motivacionales o intrínsecos están parcialmente presentes o en definitiva ausentes puede generarse en el trabajador una situación de insatisfacción a pesar de que pudieran estar cubiertas las necesidades por la presencia de los factores extrínsecos o higiénicos (altas remuneraciones, mejores beneficios, condiciones óptimas de trabajo, entre otras). Esta situación paradójica explicaría cómo en algunas profesiones altamente retadoras - de gran estima y reconocimiento social pero históricamente mal remuneradas y desempeñadas muchas veces en ambientes y condiciones hostiles o no apropiadas-, se reflejan poca rotación y altos niveles de compromiso. Es el caso de profesiones como las de médico, trabajador social y docentes u oficios como los de bombero, rescatistas, entre otros oficios que ameritan mucho compromiso vocacional para su desempeño.

\section{METODOLOGÍA}

La recolección de información se llevó a cabo a través de una encuesta on line dirigida a docentes activos y de aula de educación primaria y secundaria del sector oficial de la educación venezolana. La encuesta estaba estructurada en tres segmentos, uno destinado a recoger información sociolaboral como sexo, edad, años de servicio y titulación; el segundo segmento estuvo destinado a la escala de satisfacción laboral y el tercero a recopilar información sobre intención de renunciar al magisterio e intención de migrar de Venezuela.

La muestra definitiva estuvo conformada por 636 sujetos que cumplieron con el criterio preestablecido para formar parte de ella. Es importante destacar que el instrumento fue enviado vía on line a las bases de datos de la Federación Venezolana de Maestros (FVM), de la Fundación para el Desarrollo Integral del Docente (FUDEIND), fundación asociada a la Federación Venezolana de Maestros, y de la Editorial Santillana, sede Venezuela. La información se recopiló durante los meses de noviembre y diciembre de 2018.

La distribución de la muestra por regiones fue la siguiente:

Tabla 1. Distribución de la muestra por regiones agrupadas

\begin{tabular}{l|c|c}
\hline \multicolumn{1}{c|}{ Regiones político-administrativas agrupadas } & Sujetos & $\mathbf{\%}$ \\
\hline Capital y Central & 291 & 45,7 \\
Los Llanos & 213 & 33,4 \\
Nororiental, Insular y Guayana & 66 & 10,3 \\
Los Andes, Centroccidente y Zulia & 66 & 10,3 \\
\hline Total & $\mathbf{6 3 6}$ & $\mathbf{9 9 , 7}$ \\
\hline
\end{tabular}

El muestreo utilizado fue no probabilístico e incidental, vale decir, el instrumento fue enviado a las distintas bases de datos ya señaladas y dependió de la voluntad de los docentes responder y enviar el instrumento cumplimentado.

Validez, poder discriminatorio de los ítems y confiabilidad de la escala de satisfacción laboral. 
La escala sobre satisfacción laboral administrada a los docentes fue construida en 1996 y aplicada a una muestra de 750 maestros de Educación Básica del sector oficial venezolano ${ }^{19}$. Su proceso de construcción y validación está documentado en una publicación de 2007 en la cual explicamos en detalle dicho proceso $^{20}$. Posteriormente este instrumento fue replicado en la investigación de 2006 sobre una muestra de 1473 docentes que laboraban en el sector oficial de la educación ${ }^{21}$. En ambas oportunidades las pruebas de validez confirmaron que era un instrumento que recogía la información que pretendía recoger, proceso que se llevó a cabo a través de una prueba de jueces. Para la investigación, cuyos resultados se presentan en este artículo, se recurrió a una prueba de jueces con características de doble ciego con expertos en estudios en psicología industrial, confirmándose una vez más la validez de la escala.

A fin de asegurarnos de que variables como el tiempo y los cambios en el contexto no hubieran afectado la consistencia interna de los ítems de la escala se procedió a realizar pruebas de discriminación de ítems y confiabilidad. Los resultados del análisis de ítems señalaron que el valor mínimo de la varianza fue de 0,469 para el sueldo y el máximo de 4,09 para el trabajo en sí mismo. Se explica la baja varianza en el ítem referido al sueldo por realizarse la investigación en un contexto de hiperinflación y bajos salarios. En ese caso era muy difícil que hubiera mucha variabilidad en las respuestas emitidas por los encuestados. Pese a ello se corroboró un alto poder discriminatorio de los reactivos siguiendo el criterio sugerido por Garret ${ }^{22}$, Magnunson ${ }^{23}$ y Hernández, Fernández y Baptista $^{24}$, según el cual un ítem con una varianza mayor de 0,40 posee un alto poder discriminatorio.

Con respecto a la confiabilidad, se realizó una prueba piloto con 31 docentes escogidos al azar, previa administración on line de la encuesta. La idea era confirmar la confiabilidad de la escala y compararla con los resultados encontrados en las investigaciones previas. El estadístico aplicado en todos los casos fue el Alfa de Crombach para distribuciones normales.

Tabla 2. Coeficientes de confiabilidad

\begin{tabular}{c|c|c}
\hline Año de la investigación & Alfa de Crombach & Interpretación \\
\hline 1996 & 0,932 & $\begin{array}{c}\text { Altamente } \\
\text { confiable } \\
\text { Altamente } \\
\text { confiable } \\
\text { Altamente } \\
\text { confiable }\end{array}$ \\
\hline
\end{tabular}

19 Ramírez, "El docente frente a su trabajo".

20 Tulio Ramírez, “Construcción y validación de una escala para medir los niveles de satisfacción laboral de los docentes de Educación Básica en Venezuela", Investigación y Postgrado vol. XII, n. ${ }^{\circ}$ (1997).

21 Ramírez y D'Aubeterre, "Los niveles de satisfacción laboral".

22 Henry E. Garret. Estadística en psicología y educación (Barcelona: Paidós, 1983).

23 David Magnunson. Teoría de los tests (México: Trillas, 2009).

24 Roberto Hernández S., Carlos Fernández C. y Pilar Baptista L. Metodología de la investigación (México: McGraw-Hill, 2016). 
El resultado fue un Alfa de 0,93, lo que indica una alta confiabilidad ${ }^{25}$. Lo que sigue es el resultado de comparar los coeficientes de confiabilidad obtenidos en las investigaciones previas.

Estructura factorial de la escala y tratamiento de los datos

En su versión original, la escala de satisfacción laboral fue sometida a un análisis factorial de componentes principales para determinar cuáles eran los factores que mejor explicaban la satisfacción laboral de los docentes encuestados. Se identificaron un total de siete factores que agrupaban los ítems o reactivos que los saturaban mejor, eliminando de la escala aquellos que no saturaban en factores cuya varianza poco contribuía a explicar el fenómeno estudiado. El procedimiento utilizado y sus resultados fue explicado con detalle en un artículo de carácter metodológico publicado en $1997^{26}$. Este análisis se repitió con fines confirmatorios en la investigación de 2006, arrojando los mismos factores. Estos resultados más recientes fueron publicados en $2009^{27}$.

Para obtener los resultados medios de la escala general y de las diferentes subescalas o factores que la componen, se procedió a sumar los resultados de cada una de los ítems, dividiéndolos entre en el número total de ítems. Esto permitió obtener estadísticos descriptivos como la media aritmética y la desviación estándar. Sin embargo, entendemos que en el mundo de las ciencias sociales persiste la discusión acerca de la naturaleza de las escalas tipo Lickert o de rangos sumados. Aunque en las investigaciones sociológicas se ha utilizado como una escala continua, lo cual supone que existe un recorrido entre cada una de las alternativas de respuesta que se podría calcular con técnicas estadísticas para procesar variables de intervalo, para otro sector de investigadores esto sería una ficción metodológica ya que, en rigor, se trataría de una escala de naturaleza ordinal. Para efectos de esta investigación, si bien presentaremos los resultados medios de la escala general y las diferentes subescalas que la componen, nos abocaremos a presentar las frecuencias de respuestas con el fin posterior de hacer comparaciones con las frecuencias obtenidas en los estudios de 1996 y 2006.

Para obtener las frecuencias fueron recategorizadas las variables de continuas a ordinales, transformando los resultados medios obtenidos a puntuaciones ordinales. Esta transformación se realizó adosando un código ordinal a los rangos que acotaban las distancias entre una alternativa de respuesta y otra. Así, entonces, las alternativas de respuesta, los rangos que las delimitan (recorrido de los valores promedios obtenidos por alternativa de respuesta) y el nuevo valor ordinal asociado a cada alternativa de respuesta, se expresaron de la siguiente manera:

25 Ibíd.

26 Ramírez, "Construcción y validación de una escala".

27 Ramírez y D’Aubeterre, "La satisfacción laboral del maestro venezolano". 
Tabla 3. Alternativas de respuesta de la escala, recorrido del rango de respuestas según promedios obtenidos y nuevos valores ordinales por rango

\begin{tabular}{lcc}
\hline Alternativa de respuesta & Recorrido del rango & Nuevo valor ordinal \\
\hline Absolutamente insatisfecho & 1 a 1,99 & 1 \\
Insatisfecho & 2 a 2,99 & 2 \\
Medianamente insatisfecho & 3 a 3,99 & 3 \\
Ni satisfecho ni insatisfecho & 4 a 4,99 & 4 \\
Medianamente satisfecho & 5 a 5,99 & 5 \\
Satisfecho & 6 a 6,99 & 6 \\
Absolutamente satisfecho & 7 & 7 \\
\hline
\end{tabular}

Este mismo criterio se aplicó para el tratamiento de los datos de las diferentes subescalas que identifican los ítems asociados a cada uno de los factores identificados a partir del análisis factorial exploratorio realizado en las investigaciones anteriores.

\section{RESULTADOS}

Los docentes que conformaron la muestra definitiva, en un total de 636, presentaron edades que iban desde los 23 años hasta los 59 años, con una media de 44,6 años y una desviación típica de más o menos 8,5 , siendo el $68,9 \%$ mujeres y el 31,1 \% hombres. El nivel académico osciló entre un 39,8 \% que manifestó poseer títulos de profesor o licenciado, y un 60,2 \% un título de postgrado, lo cual da cuenta de un nivel elevado de formación académica. En cuanto a los años de servicio, el 23,7 \% manifestó tener menos de 10 años de servicio, mientras que el 76,3\% más de 10 años en el ejercicio docente. Se destaca que ninguno de los 636 docentes que contestaron el instrumento manifestó estar jubilado. Este último dato es importante ya que la condición de jubilado puede distorsionar la información suministrada ya que, al no estar activo, la percepción sobre los factores estudiados puede estar más informada o mediatizada por recuerdos de situaciones pasadas que por la experiencia directa.

Niveles de satisfacción laboral para el año 2018. Resultados medios

La escala aplicada constó de siete alternativas de respuesta, que iban desde absolutamente insatisfecho hasta absolutamente satisfecho, teniendo la primera el valor de 1 y la última el valor de 7 . Entre ellas, en un continuum, se encontraban las siguientes alternativas y sus respectivos valores: insatisfecho, medianamente insatisfecho, ni insatisfecho ni satisfecho, medianamente satisfecho, satisfecho. Se construyó con 7 alternativas de respuesta con el fin de que el docente encuestado pudiese reflejar, con una mayor comodidad y amplitud, su opinión frente al reactivo. Los resultados medios de la escala general y las subescalas fueron los siguientes: 
Tabla 4. Media y desviación típica de escala general y subescalas

\begin{tabular}{lccccc}
\hline \multicolumn{1}{c}{ Nivel de satisfacción } & Media & $\begin{array}{c}\text { Desviación } \\
\text { típica }\end{array}$ & Mínimo & Máximo & $\mathrm{N}$ \\
\hline Escala general & 3,0485 &, 98470 & 1 & 6,13 & 636 \\
Beneficios laborales & 2,2887 & 1,12302 & 1 & 6,40 & 636 \\
$\begin{array}{l}\text { Condiciones de trabajo } \\
2,4780\end{array}$ & 1,12651 & 1 & 6,29 & 636 \\
$\begin{array}{l}\text { Reconocimiento a la } \\
\text { labor }\end{array}$ & 3,8428 & 1,43790 & 1 & 7 & 636 \\
Ambiente de trabajo & 3,8042 & 1,27046 & 1 & 7 & 636 \\
Remuneraciones & 1,5409 & 1,00039 & 1 & 7 & 636 \\
Trabajo en sí mismo & 4,0554 & 1,43713 & 1 & 7 & 636 \\
Autonomía funcional & 3,3365 & 1,66459 & 1 & 7 & 636 \\
\hline
\end{tabular}

De acuerdo con los rangos descritos en la tabla 3, la escala general presenta una media de 3,04 con una desviación típica de 0,98, indicativo de un nivel de satisfacción laboral considerado como "medianamente insatisfecho". Una lectura unilateral de este resultado puede ocultar los altos niveles de malestar e insatisfacción con respecto al trabajo docente en Venezuela.

Si se observan los resultados medios y desviaciones en factores como remuneraciones $(1,54 ; 1)$, beneficios laborales $(2,28 ; 1,12)$ y condiciones de trabajo $(2,47 ; 1,12)$, se evidencia un alto grado de insatisfacción. Sin embargo, otro de los factores higiénicos, el referido al ambiente de trabajo, no presenta una media aritmética en extremo baja $(3,8 ; 1,27)$, esto lo ubica en el rango de medianamente insatisfecho. Los resultados de este último factor no ocultan el amplio descontento de los educadores entrevistados. Estos factores higiénicos, de acuerdo con Herzberg et $a l .^{28}$, si bien pueden generar insatisfacción con el entorno del trabajo, no siempre producen insatisfacción con la tarea, lo que no necesariamente supone un disparador para tomar decisiones radicales en cuanto a renuncias.

Pero la pregunta es la siguiente: ¿los factores motivadores están cumpliendo su función de mantener el compromiso con la tarea? La respuesta que se desprende de los resultados es que, definitivamente, no. Aunque el factor trabajo en sí mismo, cuya media y desviación $(4,05 ; 1,43)$ lo ubican en el rango intermedio de "ni satisfecho ni insatisfecho", las medias y desviaciones del resto de estos factores motivadores se ubica en el rango "medianamente insatisfecho". Esto deja entrever que el reconocimiento a la labor $(3,84 ; 1,43)$ y la autonomía funcional $(3,33 ; 1,66)$, factores muy ligados a la pasión y vocación por el trabajo, evidencian señales preocupantes de insatisfacción laboral en los docentes venezolanos.

Niveles de satisfacción laboral para el año 2018. Resultados porcentuales

Una vez seguido el procedimiento descrito en el apartado Estructura factorial de la escala y tratamiento de los datos, se calcularon las frecuencias de respuestas para la escala general y las diferentes subescalas que la estructuraban.

28 Herzberg, Mausner y Snyderman, The Motivation To Work. 
Con el fin de simplificar el análisis se procedió a sumar los porcentajes obtenidos en las alternativas de respuesta de cada reactivo de la escala. Los resultados que identifican un estado de insatisfacción de los docentes entrevistados (AI+I+MDI), revelan que el 84,9\% manifiestan su insatisfacción sobre la labor docente que realizan. Este resultado es un indicador que podría explicar, en buena parte, el descontento y desmotivación manifiesta de este sector laboral, evidenciado en los continuos conflictos gremiales que ha experimentado este sector en los últimos diez años en Venezuela.

Las frecuencias acumuladas de las alternativas "altamente insatisfechas, insatisfechas" y "medianamente insatisfecho", para los factores beneficios laborales, condiciones de trabajo y remuneraciones, están entre el 89,9\% y el 95,8\%. Solo 1 de cada 10 entrevistados no se ubica en esas categorías. En este grupo de factores higiénicos, solo el factor ambiente de trabajo es percibido de manera insatisfactoria por la mitad de los entrevistados (49,2\%).

Del grupo de los factores motivadores (los que explican el afecto por el trabajo a pesar del descontento generado por los factores higiénicos), encontramos que generan sentimientos de insatisfacción para un sector del grupo estudiado que representa entre el $41 \%$ y el 58,4\%, lo cual no es una cifra despreciable.

Ahora bien, es necesario comparar estas frecuencias con las obtenidas en los estudios anteriores con el fin de obtener pistas sobre las tendencias de satisfacción o insatisfacción sobre los factores estudiados a lo largo de 24 años.

Niveles de satisfacción laboral del docente venezolano (1996, 2006 y2018)

El gráfico 1 muestra los resultados porcentuales de los niveles de satisfacción laboral sobre los siete factores que integraron la escala de satisfacción laboral construida y validada en el año 1996. Se presentan, para efectos de comparación analítica, los resultados obtenidos en 1996, 2006 y 2018. 
Gráfico 1. Niveles diferenciales de satisfacción laboral (1996, 2006, 2018)

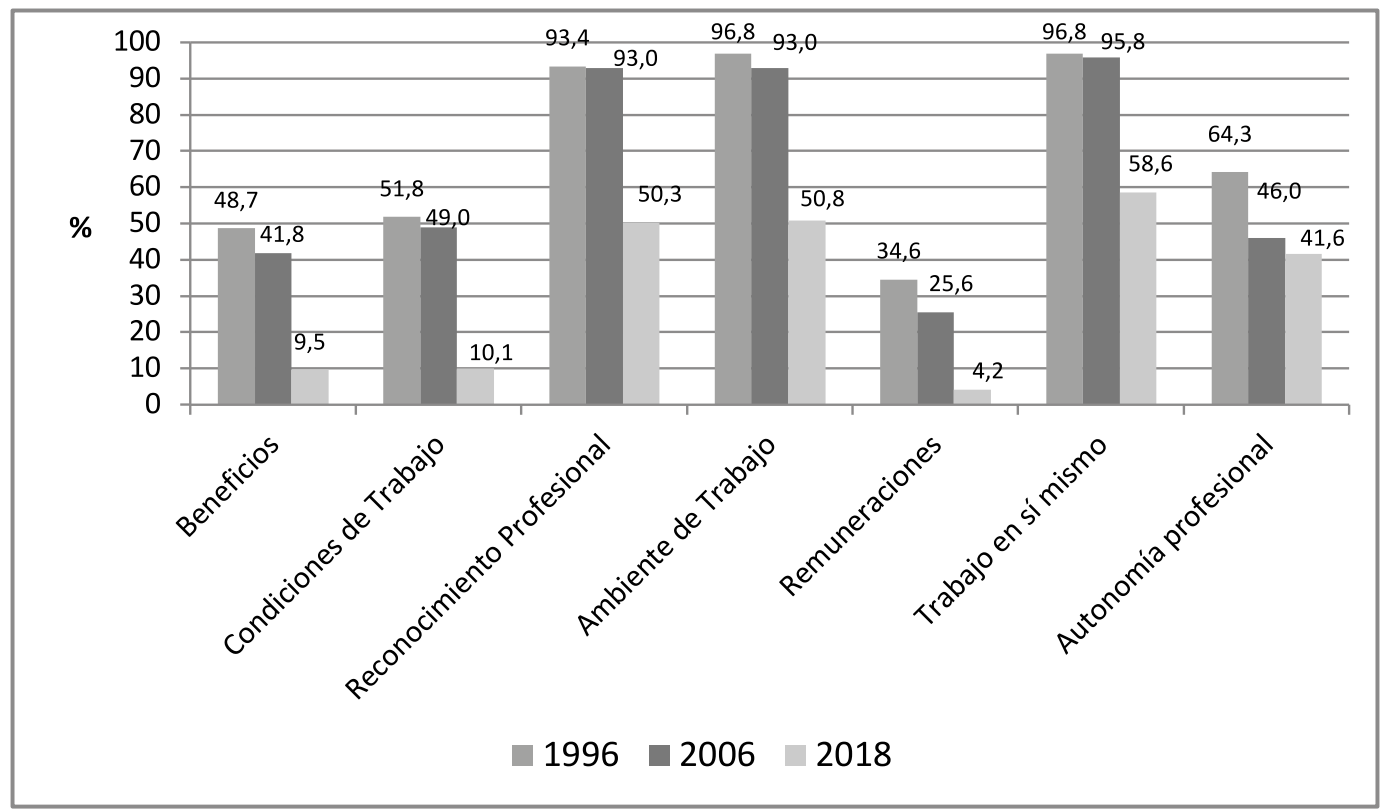

Con el fin de hacer una interpretación de estos resultados lo más ordenada posible, se procederá a agrupar los factores de acuerdo con su naturaleza. Se comenzarán a analizar los resultados referidos a los llamados factores higiénicos.

En cuanto a los beneficios laborales, los niveles de satisfacción han venido disminuyendo de manera continua a través de los últimos 24 años. Para 1996, el 48,7 \% manifestó estar satisfecho; para el 2006 esta percepción bajó en 7 puntos porcentuales, ubicándose en el 41,8\%; para 2018 solo el 9,5\% de la muestra mostró un alto nivel de satisfacción. Esto muestra cómo, en 24 años, se han deteriorado los beneficios que por derecho corresponden a los docentes del sector oficial. Los reactivos que corresponden a este factor son: los beneficios obtenidos por el IPASME (25); cumplimiento de los compromisos administrativos por parte del Ministerio de Educación (26); los logros de los contratos colectivos (27); la actuación de los gremios docentes (28); la atención recibida por el Ministerio de Educación y Deportes (31).

En cuanto al factor condiciones de trabajo, los niveles de satisfacción rondaban el $50 \%$ tanto en 1996 como en 2006, lo cual reflejaba cierta estabilidad en la opinión. Sin embargo, para 2018 esta percepción se redujo en casi 40 puntos porcentuales. Solo un $10 \%$ de la muestra manifestó estar satisfecho con el factor. Al igual que el factor beneficios laborales, las condiciones de trabajo de los docentes han tenido un pronunciado nivel de deterioro. Los ítems que conformaron este factor son: la dotación de mobiliario (1); los incentivos recibidos (2); las condiciones de infraestructura del centro de trabajo (13); la limpieza y mantenimiento del plantel (16); la disponibilidad de recursos instruccionales (19); la supervisión (20); las oportunidades de formación (21). 
El factor ambiente de trabajo fue uno de los 3 factores que en el estudio realizado en 1996 obtuvo valores de satisfacción por encima del 90 \%; incluso en el estudio realizado en 2006, aunque bajaron un poco, esos niveles no se estuvieron por debajo del $90 \%$. Sin embargo, para 2018, solo el 50,8 \% de los entrevistados manifestó satisfacción por el factor. Se constata un descenso de casi 40 puntos porcentuales, lo cual lo hace uno de los factores que mayor perdió popularidad entre los docentes. Este factor estaba conformado por los siguientes reactivos: la accesibilidad al centro de trabajo (17); la disposición, hábitos y actitudes de los estudiantes (22); las relaciones con los padres y representantes (23); la realización personal conseguida en el trabajo (24); las relaciones entre los compañeros de trabajo (29); las relaciones entre los docentes y los directivos (30).

Como era de esperarse, el nivel de satisfacción sobre el factor remuneraciones nunca fue de los más altos en popularidad. Los docentes venezolanos, al igual que los docentes de la región, nunca han recibido altos niveles de remuneración. Al comparar con los salarios de otros profesionales de la administración pública se puede evidenciar la razón. No más del $35 \%$ de los docentes manifestó en 1996 estar satisfecho con este factor; en 2006 este porcentaje bajó a 25,6 \% y en 2018 se redujo a 2,6\%. Dicho de otra manera, para 2018 más del $90 \%$ de los docentes entrevistados manifestó inconformidad con este factor. El deterioro de las remuneraciones es uno de los aspectos más sensibles, más en un país con una economía hiperinflacionaria. Según la Asamblea Nacional, la inflación acumulada para 2018 fue de $1698488 \%{ }^{29}$. Los ítems que componen este factor son: el salario (14); primas y bonos (15); ingresos versus responsabilidades laborales (18).

Con respecto a los llamados factores motivadores (aquellos cuya ausencia genera insatisfacción con la tarea), los resultados diferenciales fueron los que a continuación se comentan.

El reconocimiento a la labor profesional es uno de los factores que más opinión favorable obtuvo en el estudio de 1996 (93,4 \%). Para 2006, las expresiones de satisfacción bajaron en solo cuatro décimas, ubicándose en $93 \%$. Para el estudio de 2018, esa percepción bajó en casi un 50 \%, se ubicó en 50,3\%. Los ítems que la informan son los siguientes: el reconocimiento del trabajo por parte de los directivos (5); de los alumnos (6); de los padres o representantes (10); de los compañeros de labores (11). A nuestro entender, que los docentes no se sientan satisfechos con el reconocimiento recibido por directivos, colegas, alumnos y padres y representantes. Si algo enorgullecía al maestro era el reconocimiento social de su profesión. Este es un elemento coadyuvante al estado de desmotivación que conduce al ausentismo y, eventualmente, a las renuncias.

El factor trabajo en sí mismo se refiere a la naturaleza del trabajo desempeñado, y en el caso que nos ocupa, a la labor docente estrictamente dicha. Alude más al plano vocacional, al compromiso con lo que se hace que a la destreza. Los estudios de 1996 y 2006 evidenciaron que más del 95 \% de los entrevistados

29 C. Seijas, "Venezuela cerró 2018 con una inflación de casi 1.700.000\%”, El Nacional, Caracas, 10 de enero, 2019. 
mostraban un alto nivel de satisfacción con la labor desempeñada. Para 2018, los datos arrojan un descenso al 58,6\%, lo cual supone que 6 de cada 10 docentes se encuentran satisfechos y motivados por su trabajo. De seguir esta tendencia tendremos cada vez más docentes asumiendo la tarea pedagógica más como un trabajo que puede ser temporal y no como una razón de vida y de servicio al prójimo. Los ítems que conformaron en la escala aplicada estos factores son: tareas y actividades realizadas en el trabajo (7); la carga de trabajo (8); la calidad del trabajo realizado (9); la relación trabajo y expectativas profesionales (12).

Una de las características que diferencia a las profesiones de los oficios es la autonomía funcional o la capacidad de decidir autónomamente con base en criterios teóricos y técnicos adquiridos a través de estudios formalmente realizados a nivel universitario, las mejores opciones o protocolos para el desempeño de la labor profesional. Esa capacidad es lo que informa el factor autonomía funcional. En el estudio realizado en 2006 se observó con cierta alarma que la cantidad de docentes satisfechos con este factor se había reducido en 18 puntos porcentuales $^{30}$. La alarma se debía a que esta puntuación expresaba el descontento cada vez mayor de los docentes sobre las políticas intervencionistas y controladores por parte del Ministerio del Poder Popular para la Educación a través de directivos, supervisores y comisarios políticos miembros de los llamados consejo comunales, sobre los docentes de las escuelas del sector oficial, así como la presión sobre los llamados "Docentes Interinos", quienes fueron obligados a ser sumisos al gobierno a riesgo de ser despedidos de sus cargos. Para 2018, el porcentaje de los docentes satisfechos con este factor descendió a 41,6\%. Los ítems que integran este factor son: autonomía del docente en el trabajo (3) y para introducir cambios (4).

\section{CONCLUSIONES}

Monitorear el estado de ánimo del trabajador debe ser una política permanente de recursos humanos en cualquier organización. Estos estudios periódicos permiten diagnosticar y dar explicación sobre problemas que pudiesen estar afectando la productividad, el compromiso con el trabajo y con la organización, la asistencia, la motivación, la calidad del trabajo, la eficiencia, la paz laboral, el ambiente de trabajo, la permanencia en el empleo y tantas otras variables que condicionan el clima laboral en la organización. Estas consideraciones no son exclusivas para el mundo empresarial, comercial y de servicios del sector privado, donde el permanente riesgo de perder la inversión obliga a atajar a tiempo todo aquello que afecte la razón de ser de la empresa privada, a saber, generar ganancias a cambio de una oferta de productos o servicios de calidad. Por el contrario, también se debe monitorear el estado de ánimo del trabajador

30 Ramírez y D’Aubeterre, “Los niveles de satisfacción laboral”. 
del sector público, aunque esta no sea precisamente la tradición ni en Venezuela ni en el resto de América Latina.

Los diagnósticos sobre la satisfacción del trabajador del sector público con la labor que desempeña tienen la misma importancia que los que se realizan en el sector privado. No solo hay que evitar el despilfarro de los recursos públicos sino tratar de garantizar que el servicio prestado sea eficiente y de calidad. Al final, los beneficiarios son los ciudadanos que con sus impuestos ayudan a alimentar los presupuestos públicos que financian la burocracia, los profesionales, técnicos y obreros, así como la infraestructura, dotación y organizaciones gubernamentales que han de prestar el servicio. Las huelgas, paros, inasistencias injustificadas, la falta de compromiso y una alta rotación de personal suele ser producto del descontento que genera un ambiente de trabajo hostil y desmotivador.

El sistema educativo dependiente del sector oficial no está exento de padecer los mismos males que pueden aquejar al trabajador de la empresa privada. Algo tan caro para el desarrollo de un país como es la educación, no puede permitirse el lujo de desatender el menor síntoma de malestar de sus docentes. A través del ministerio del ramo los gobiernos deben realizar estudios cada cierto tiempo para conocer hasta dónde asciende la temperatura del descontento de los docentes, en lugar de relegar esta responsabilidad a los gremios o sindicatos. Hay que entender que un adecuado ambiente de trabajo, una remuneración acorde con la importancia del trabajo desempeñado y las credenciales académicas, el reconocimiento a la labor cumplida, condiciones adecuadas para desarrollar el proceso de enseñanza y el respeto a las decisiones pedagógicas para proveer una educación de calidad, garantizarán un ambiente laboral proclive al pleno desarrollo de la vocación magisterial y a un aumento del nivel de compromiso por impartir una educación cada vez mejor.

A pesar de la falta de estadísticas oficiales que lo evidencien la educación venezolana no está pasando por su mejor momento. Las constantes denuncias de falta de docentes en asignaturas científicas del pénsum de estudios, el elevado ausentismo por parte de los docentes, las renuncias intempestivas a mitad del año escolar, son señales del evidente descontento en el mundo magisterial. Los resultados presentados en este informe de investigación así lo demuestran. La comparación de resultados en los tres estudios realizados en un espacio de 24 años dejan en claro que la insatisfacción de los docentes venezolanos va in crescendo, sin que se evidencie alguna posibilidad de revertir esa tendencia, de mantenerse o profundizarse el deterioro de las condiciones de trabajo.

A nuestro entender, lo más preocupante que revelan los datos presentados es que cada vez hay más docentes insatisfechos con los factores motivadores. Hagamos un ejercicio comparativo con los resultados de los tres estudios.

Si se observan las cifras de 1996 y 2006, más del $90 \%$ de los docentes entrevistados señalaron satisfacción sobre factores como el reconocimiento a la labor cumplida; el ambiente de trabajo y el trabajo en sí mismo; inclusive casi un $70 \%$ lo hizo sobre la autonomía para el desempeño del trabajo. Esto comprobaría la 
tesis de Herzberg y colaboradores ${ }^{31}$ que señala que, aun habiendo descontento o insatisfacción sobre los factores higiénicos (remuneraciones, condiciones de trabajo, beneficios y ambiente de trabajo), si se mantienen niveles de satisfacción sobre los factores motivadores, la probabilidad de conflictos labores, renuncias masivas y ausentismo, se reduce considerablemente. Esto explicaría por qué en Venezuela la docencia fue una de las actividades más estables. Los maestros y profesores nunca estuvieron bien remunerados y las condiciones de trabajo no eran las más acordes, pero la vocación y el compromiso con el magisterio compensaban cualquier descontento. De allí que la rotación de personal no era lo que caracterizaba a ese sector laboral.

Para el año 2018 esta situación cambia de manera radical. Los niveles de satisfacción sobre los factores higiénicos disminuyen vertiginosamente en razón de la severa crisis económica que ha venido sufriendo Venezuela. Esta crisis se acentuó desde el año 2012 con la consolidación de un modelo que ha deteriorado severamente los indicadores económicos, incidiendo en la merma de la capacidad adquisitiva del salario, una hiperinflación catalogada como la más alta del mundo, una maxidevaluación de la moneda, la reducción de las exportaciones petroleras y la casi extinción del aparato productivo. Esta situación de precariedad del poder adquisitivo del salario, las cada vez peores condiciones de trabajo por la falta de mantenimiento en las escuelas, la inexistente dotación de recursos, el control político a través de resoluciones ministeriales como la 058 que permite el acceso de los Consejos Comunales (organizaciones tuteladas desde el gobierno nacional) a las escuelas para participar en la toma de decisiones, la presión sobre los docentes para que participen de manera obligada en actividades de apoyo al gobierno chavista, el enrarecido ambiente de trabajo por la conflictividad política y el escaso reconocimiento a los profesionales de la docencia, conforman un contexto que podría ser la causa de los extremadamente bajos niveles de satisfacción que arrojan los resultados de esta investigación.

La preocupante conclusión que se desprende de estos resultados apunta hacia un futuro muy comprometido para la educación venezolana. De seguir esta tendencia de descontento en el sector docente del país, no solo se verificarán altos niveles de renuncias, sino pocas posibilidades de que las nuevas generaciones opten por seguir la carrera docente. Ya se está evidenciando lo primero y, en cuanto a lo segundo, las cifras de aspirantes a estudiar la carrera docente son cada vez menores. Cada día es más difícil conseguir profesores de Química, Biología, Matemática, Física, entre otras asignaturas. Se debe revalorizar la educación y por ende el trabajo docente. Eso amerita un cambio de perspectiva que pasa por asumir a la educación como la clave del desarrollo y no como la oportunidad para ideologizar en torno a un determinado proyecto político, tal como está taxativamente planteado en el llamado Plan de la Patria 2013-2019, hoja de ruta del gobierno socialista, el cual señala en el numeral 2.4.1.1, que entre los objetivos estratégicos en materia educativa se debe "Adecuar los planes

31 Herzberg, Mausner y Snyderman, the Motivation to Work. 
de estudio en todos los niveles para la inclusión de estrategias de formación de valores socialistas y patrióticos" ${ }^{\prime 32}$. Es una doctrina supremacista muy alejada del ideal de la educación democrática, la cual debe estar fundamentada en el respeto a todas las corrientes de pensamiento, tal como lo señala el artículo 102 de la Constitución Nacional de la República Bolivariana de Venezuela.

\section{BIBLIOGRAFÍA}

Barry, J. "Satisfaction in job". Cornell review 3, n. ${ }^{\circ}$ (1985): 12-23.

Blum, Milton I. y James C. Naylor. Psicología industrial: Sus fundamentos teóricos y sociales. Ciudad de México: Trillas, 1990.

Byars, Lloyd y Leslie W. Rue. Administración de recursos humanos. Conceptos y aplicaciones. México: Nueva Editorial Interamericana, 1983.

Cabrera, Carlos E., Sergio Franco, Guillermo González, Guadalupe Vega, Jaime Parra y Jorge Íñiguez. "Satisfacción laboral de profesionales de la salud en el IMSS, Jalisco 1999-2002". Revista Médica IMSS 42, n. ${ }^{\circ} 3$ (2003): 193-198.

Flippo, Edwin B. Principios de administración de personal. México: McGraw-Hill, 1982.

Gamero, Carlos. Análisis macroeconómico de la satisfacción laboral. Madrid: Consejo Económico y Social, 2005.

Garret, Henry E. Estadística en psicología y educación. Barcelona: Paidós, 1983.

Hernández S., Roberto, Carlos Fernández C. y Pilar Baptista L. Metodología de la investigación. México: McGraw-Hill, 2016.

Hackman, Richard J. y Edward E. Lawler. "Employee reactions to job characteristics". Journal of Applied Psychology 55, n. ${ }^{\circ} 3$ (1978): 259-286.

Herzberg, Frederick, Bernard Mausner y Barbara Bloch Snyderman. The Motivation To Work. New York: Wiley, 1959.

Lawler, Edward E. y Lyman W. Porter. "What job attitudes tell about motivation”. Harvard Business Review 46, n. ${ }^{\circ} 1$ (1969): 118-126.

Ley del Plan de la Patria. Segundo Plan Socialista de Desarrollo Económico y Social de la Nación 2013-2019. Gaceta Oficial de la República Bolivariana de Venezuela, n. ${ }^{\circ} 6.118$ Extraordinario, 4 de diciembre de 2013.

Magnunson, David. Teoría de los tests. México: Trillas, 2009.

Mc Gregor, Douglas. El aspecto humano de las empresas. México: Diana, 1972.

Maslow, Abraham H. Motivation and Personality. New York: Harper and Row, 1954.

Maslow, Abraham H. El management según Maslow: Una visión humanista para la empresa de hoy. Barcelona: Editorial Paidós Ibérica, 2005.

Merino, Enrique. "Teoría del ajuste laboral y orientación vocacional”. International Journal of Developmental and Educational Psychology 5, n. ${ }^{\circ} 1$ (2011): 529-535.

Perry, John. Las relaciones humanas en la industria. Buenos Aires: Editorial Selección Contable, 1961.

Ramírez, Tulio y María E. D’Aubeterre. “La satisfacción laboral del maestro venezolano. Un abordaje a partir del análisis de componentes principales". En La enseñanza desde la participación de los actores. Hacia una escuela innovadora, editado por Carlos Manterola y Gilberto Graffe. Caracas: CIES-Universidad Central de Venezuela, 2009.

Ramírez, Tulio, María E. D’Aubeterre y Juan C. Álvarez. “Un estudio sobre estrés laboral en una muestra de maestros de Educación Básica del área metropolitana de Caracas". ExtraMuros, n. 29 (2009): 69-99.

32 Ley del Plan de la Patria. Segundo Plan Socialista de Desarrollo Económico y Social de la Nación 2013-2019. Gaceta Oficial de la República Bolivariana de Venezuela n. ${ }^{\circ} 6.118$ Extraordinario, 4 de diciembre de 013, 14. 
Ramírez, Tulio, María E. D’Aubeterre y Juan C. Álvarez. “Percepción de estresores en profesores universitarios venezolanos. Un estudio descriptivo". Akademus 14, n. ${ }^{\text {os }} 1$ y 2 (2012): 113-138.

Seijas, C., “Venezuela cerró 2018 con una inflación de casi 1.700.000\%”, El Nacional, Caracas, 10 de enero, 2019.

\begin{tabular}{l|l|} 
& \\
\hline Cómo citar: & \\
Ramírez, Tulio; Torres Cruz, Doris Lilia. “Una lectura comparativa & \\
sobre el nivel de satisfacción laboral de los docentes venezolanos \\
entre 1996 y 2006”. Revista Historia de la Educación Latinoamericana. \\
22 No. 35 (2020): 253-272 \\
https://doi.org/10.19053/01227238.11969 \\
\begin{tabular}{|l} 
@C)@@ Esta obra está bajo una licencia Creative Commons. \\
Reconocimiento-No Comercial-Sin Obra Derivada 2.5 Colombia.
\end{tabular} \\
\hline
\end{tabular}

